Case Report

\title{
Minimally Invasive Resin-Bonded Inlay-Retained Cantilever Fixed Dental Prosthesis: A Clinical Report
}

\author{
Albandari Bin-Rubayan $\mathbb{D}^{1},{ }^{1}$ Abdulaziz Samran $\mathbb{D}^{2,3}$ and Ali Alqerban $\mathbb{D}^{4,5}$ \\ ${ }^{1}$ Ministry of Health (SCFHS), Prosthodontics Program (Training Center: Department of Restorative and Prosthetic Dental Sciences, \\ College of Dentistry, Dar Al Uloom University), Riyadh, Saudi Arabia \\ ${ }^{2}$ Department of Restorative and Prosthetic Dental Sciences, School of Dentistry, Dar Al-Uloom University, Riyadh, Saudi Arabia \\ ${ }^{3}$ Department of Prosthodontics, School of Dentistry, Ibb University, Yemen \\ ${ }^{4}$ Department of Preventive Dental Sciences, College of Dentistry, Prince Sattam Bin Abdulaziz University, Alkharj, Saudi Arabia \\ ${ }^{5}$ Department of Preventive Dental Science, Dar Al Uloom University, Riyadh, Saudi Arabia
}

Correspondence should be addressed to Abdulaziz Samran; asamran@dau.edu.sa

Received 6 January 2021; Accepted 11 May 2021; Published 30 May 2021

Academic Editor: Asja Celebic

Copyright (c) 2021 Albandari Bin-Rubayan et al. This is an open access article distributed under the Creative Commons Attribution License, which permits unrestricted use, distribution, and reproduction in any medium, provided the original work is properly cited.

\begin{abstract}
This clinical report demonstrates the treatment of a healthy adult patient referred for prosthodontic treatment after orthodontic treatment with a resin-bonded inlay-retained cantilever fixed dental prosthesis (IRCFDP). The purpose of this report was to demonstrate the esthetic, functional, and conservative properties of a resin-bonded IRCFDP fabricated from monolithic zirconia which can be placed in posterior area in certain situations. Acceptable esthetics with a conservative and functional result were achieved by using a resin-bonded inlay-retained cantilever fixed dental prosthesis (IRCFDP). All laboratory and clinical procedures of this case report are described.
\end{abstract}

\section{Introduction}

Replacement of missing teeth area can be accomplished with resin-bonded fixed dental prostheses (FDPs), conventional fixed dental prostheses (FDPs), implant-supported FDPs, or removable dentures. Conventional FDPs typically require the removal of $50 \%$ to $70 \%$ of sound dental tissue $[1,2]$ which has been reported to lead to damage tooth vitality in $10 \%$ of the FDPs $[3,4]$. For that reason, these options are indicated when the adjacent teeth are extensively restored or damaged. Tooth structure can be preserved with adhesively placed resin-bonded FDPs.

Metal resin-bonded fixed dental prostheses (RBFDPs) have been recommended as a conservative option to conventional FDPs [5-8]. However, when a 2-retainer design is used, debonding of one abutment often results in caries that is not perceived by the patient. Ceramic anterior RBFDPs were first described in the 1990s and have been used to replace posterior teeth in certain situations [9]. Ceramic cantilever RBFDPs were suggested by Kern et al. [10] since their clinical study of ceramic RBFDPs with two retainers exhibited high fracture rates within the first year after insertion. The cantilever design leads to reduced shear and tensile forces compared with splinting two abutments teeth with differential movement [11]. In addition, when cantilever RBFDPs debond, the patient will immediately notice [12]. In a report of 10-year follow-up study, the survival rate of cantilever ceramic RBFDPs was 98.2\% [13], which was better than that of tworetainer RBFDPs [14]. A zirconia inlay-retained fixed dental prosthesis (IRFDP) design was suggested by Wolfart and Kern [15], and Bishtia et al. [16] tested a new design for inlayretained cantilever fixed dental prostheses (IRCFDPs) in an in vitro study. This design represents a conservative solution which can be applied in some patients, such as those with existing restorations or caries and when an implant-supported prosthesis is contraindicated. The aim of this clinical report was to 
describe the replacement of a missing mandibular right first molar with a computer-aided design and computer-aided manufacture (CAD-CAM) resin-bonded IRCFDP made of zirconia ceramic.

\section{Case Report}

A healthy 42-year-old man visited the Department of Prosthodontics of Dar Aluloom University seeking a replacement for his missing mandibular right first molar which had been extracted several years ago after unsuccessful endodontic treatment (Figure 1). His dental history also included orthodontic treatment for about two years to correct malocclusion (crowding in some areas and spacing in other areas because of his missing four first molars). The orthodontist referred him to the prosthodontic clinic to restore the narrow space in the mandibular right first molar area, which had not been closed by the orthodontic treatment. The clinical investigation revealed a narrow mandibular first molar space that contraindicated for implant placement (Figure 2). In addition, the mandibular right second molar had an occlusal amalgam restoration with occlusal recurrent caries (Figure 3). The majority of teeth were vital, and the oral hygiene was good. A periapical radiograph revealed a deep existing amalgam restoration in the mandibular right second molar, with no periapical abscesses or other significant findings. After making primary impressions for diagnostic casts and consultation with other specialists, a treatment plan was formulated to replace the missing tooth with a minimally invasive resinbonded IRCFDP [17]. The patient was informed about the risk of the proposed treatment and its alternatives, including a conventional cantilever FDP and IRFDP. After rubber dam placement, the existing amalgam restoration on the second right molar and the caries were removed, providing a cavity for the inlay retainer, which followed the preparation principles for ceramic inlay restorations [18]. The inlay cavity was prepared without bevels with fine-grit diamond rotary instruments by removing sharp margins, smoothing the pulpal floor, and preparing two retainer-wings buccally and lingually. The enamel surface was reduced by approximately $0.5 \mathrm{~mm}$ to provide a $3 \times 4 \mathrm{~mm}$ enamel area for bonding (Figure 4). The reduction was parallel to the path of insertion of the inlay retainer. After abutment preparation, polyvinyl siloxane impression material was used for the final impression (Take 1 Advanced; Kerr Corp) in a stock tray and poured with Type IV dental stone (Fujirock; GC Corp). The stone cast was scanned with a laboratory scanner. The resin-bonded IRCFDP was designed as an inlay retainer with buccal and lingual retainer wings and a second premolar pontic and milled from an A2 zirconia shade block (Cercon HT Full Contour Zirconia; Dentsply Sirona) (Figures 5 and 6).

After sintering, the framework was seated on the cast after minor corrections, and then, the marginal fit and internal fit were checked intraorally using an explorer and a silicone indicator paste (Fit Checker, GC Corp). The silicone indicator paste exhibited a homogeneous and thin thickness which was accepted. For the inlay retainer, the minimum thickness was $3 \mathrm{~mm}$, and for the buccal and lingual retainer wings, it was $0.7 \mathrm{~mm}$. The dimensions for the proximal con-

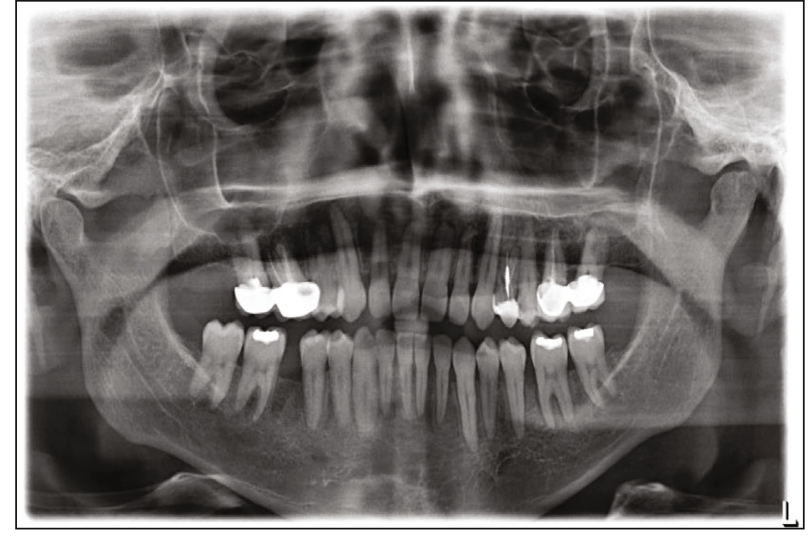

Figure 1: Pretreatment panoramic radiograph of initial patient presentation.

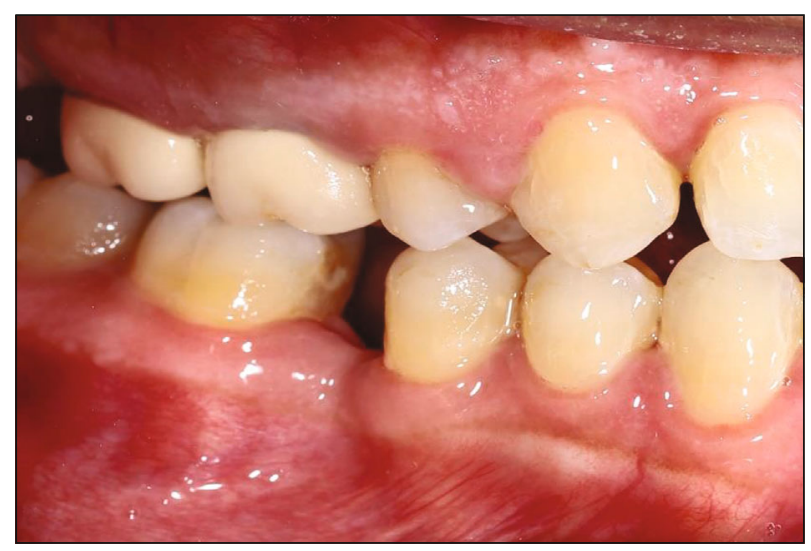

FIgURE 2: Pretreatment lateral view of mandibular arch.

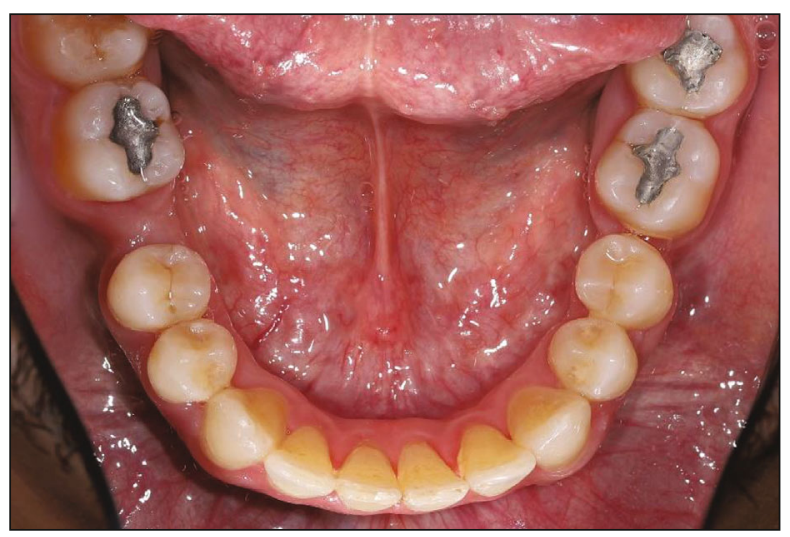

Figure 3: Pretreatment occlusal view of mandibular arch.

nector were approximately $4 \mathrm{~mm}$ in height and $4 \mathrm{~mm}$ in width. After try-in stage (Figure 7) and to remove the residues of the saliva and blood, the bonding surface of resin-bonded IRCFDP was cleaned using hot water steamer. Then, the bonding surface of the inlay retainer and the buccal and lingual retainer wings were airborne-particle abraded with $50 \mu \mathrm{m} \mathrm{Al}_{2} \mathrm{O}_{3}$ for 10 seconds with $0.1 \mathrm{MPa}$ pressure [19]. After that, the prosthesis was ultrasonically cleaned for 


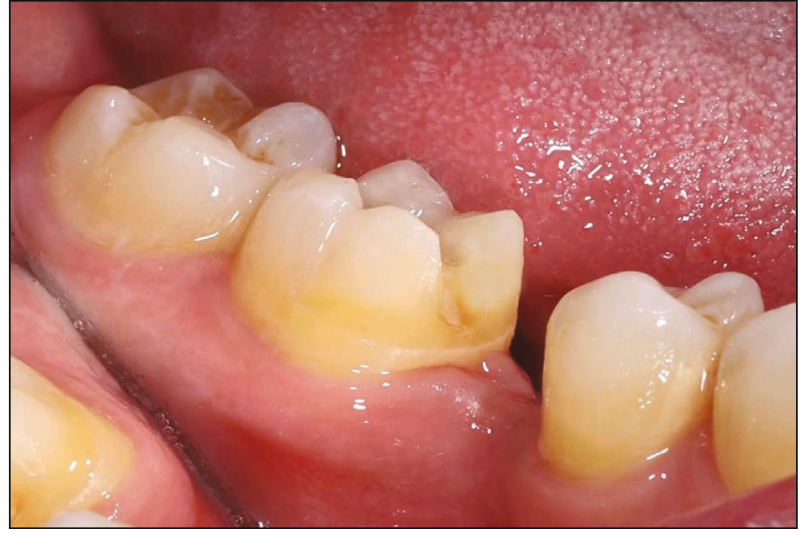

Figure 4: The mandibular right second molar after preparation.

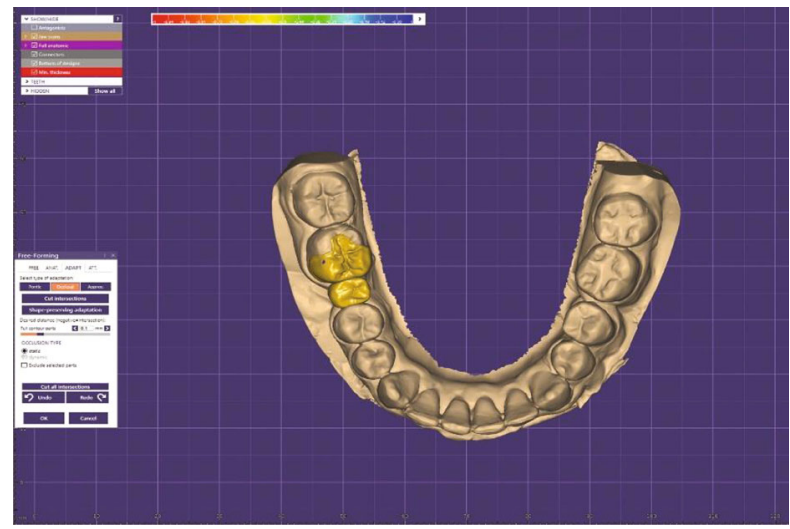

Figure 5: Computer-aided designed framework (occlusal view).

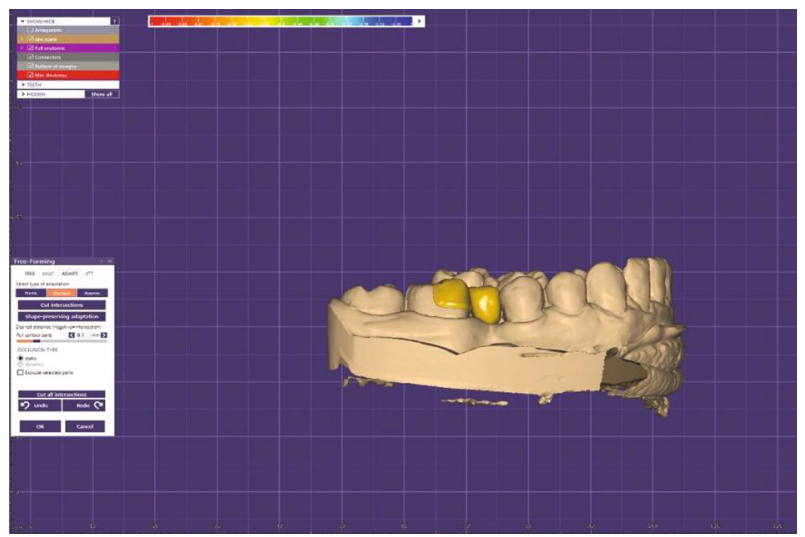

Figure 6: Computer-aided designed framework (buccal view).

3 minutes in alcohol path to remove the abrasive residues. Consequently, the resin-bonded IRCFDP was stored in a special container while the preparation surfaces of the abutment tooth are cleaned and treated for the next cementation process.

A rubber dam was applied during adhesive cementation, and the abutment tooth was cleaned with pumice. Then, the preparation surfaces of the abutment tooth were etched with a 37\% phosphoric acid gel (Cica; Promedica) for 15-30 sec (according to the tooth structure; dentin or enamel). Then, the acid etch gel was sprayed off with water for $15 \mathrm{sec}$, and

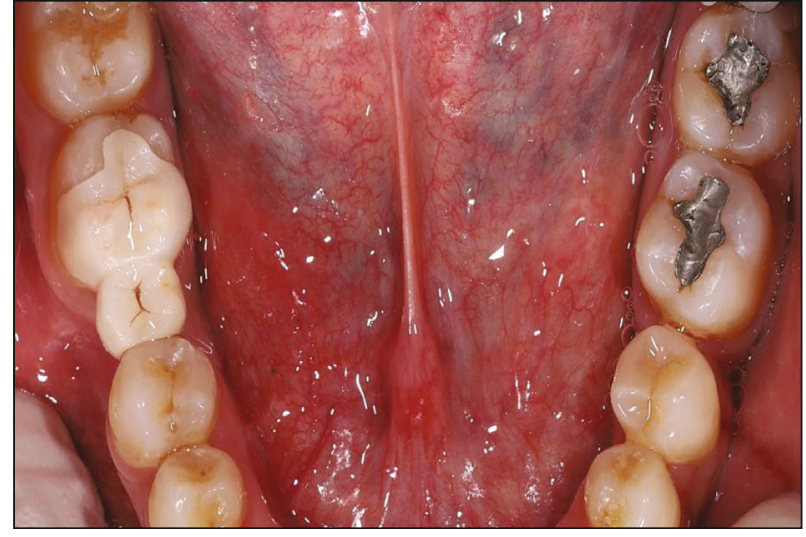

FIgURE 7: Evaluation of resin-bonded inlay-retained cantilever fixed dental prosthesis before cementation.

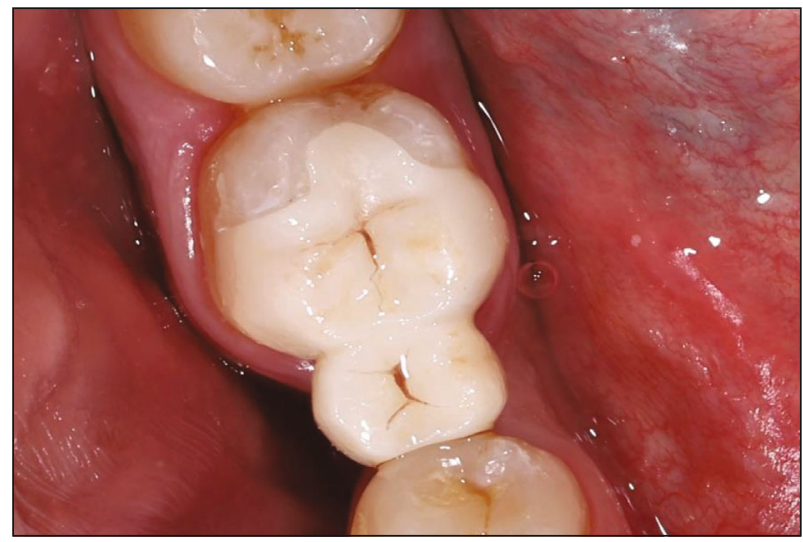

FIGURE 8: Occlusal view of resin-bonded inlay-retained cantilever fixed dental prosthesis immediately after insertion.

the tooth was thoroughly dried with air stream. After that, the enamel and dentin walls were conditioned with corresponding primer (Compobond LCM Primer; Promedica) which was mixed according to manufacturer's instructions and applied for 30 seconds before dispersing the excess using gentle oil-free air stream. Then, the adhesive material (Compobond LCM Adhesive; Promedica) was mixed according to manufacturer's instructions and applied for 15 seconds before removing the excess using gentle oil-free air stream and light cured for $15 \mathrm{sec}$. Finally, bonding surfaces of the prosthesis were primed with a ceramic primer (Aureocem DC Ceramic Primer; Promedica) using a microbrush. The primer was left for $60 \mathrm{~s}$, and the excesses were removed with an oil-free air stream. After that, the cement (Aureocem DC Automix, Promedica) was distributed over the resin-bonded IRCFDP bonding surfaces, and the prosthesis was seated in place (Figure 8). Steady finger pressure was applied during the setting time. After cementation, the function and occlusion were checked using articulator papers.

\section{Discussion}

This clinical report describes replacing the narrow space of a missing mandibular right molar with an esthetic and conservative resin-bonded IRCFDP fabricated from monolithic 
zirconia. The esthetics of this zirconia resin-bonded IRCFDP were excellent, better than the metal-ceramic FDP. Although this technique has been recommended only as an interim technique of replacing of missing teeth, however, its conservative preparation, esthetic, and reported survival rate suggest that it may be considered as a definitive treatment choice in certain situations $[6,13,20-22]$. The framework of the current resin-bonded IRCFDP was made of monolithic zirconia material based on laboratory studies that reported higher fracture load for zirconia-based IRFDPs than those made from lithium disilicate ceramic [23-25]. This resinbonded IRCFDP design was developed to overcome the high failure rate of previous IRFDP designs which might not be recommended for clinical indications [26-28]. The presence of wings in this design reduced stress on the inlay retainer caused by torsion forces applied nonaxially and to increase the enamel surface area for bonding. Similar retainer wings have been used in previous studies with cantilevered ceramic resin-bonded FDPs [20, 29].

Disadvantages of this design included the poor color match of the resin-bonded IRCFDP to the adjacent natural teeth because the prosthesis was made completely of monolithic zirconia (Figure 6). Additionally, the wings were somewhat bulky which added approximately 0.1 to $0.2 \mathrm{~mm}$ to the tooth contour. A suggested minimum thickness of 0.6 to $0.7 \mathrm{~mm}$ was used for the zirconia wings, and the tooth preparation was minimal in this area (approximately $0.5 \mathrm{~mm}$ ) [17].

\section{Conclusion}

An esthetic and conservative approach to replacing the narrow space of a missing posterior molar with a resin-bonded IRCFDP fabricated from monolithic zirconia was described. After a conservative tooth preparation, a resin-bonded IRCFDP with buccal and lingual wings was fabricated from monolithic zirconia. The patient was so satisfied and pleased with the aesthetics and functional results. This treatment option helped maintain the abutment and the adjacent teeth. Clinical follow-up is important to determine the success rate of this type of prosthesis.

\section{Conflicts of Interest}

The authors have no conflict of interest to declare.

\section{Acknowledgments}

The authors would like to thank the Deanship of Graduate Studies and Research in Dar Aluloom University for its support.

\section{References}

[1] D. Edelhoff and J. A. Sorensen, "Tooth structure removal associated with various preparation designs for posterior teeth," The International Journal of Periodontics \& Restorative Dentistry, vol. 22, no. 3, pp. 241-249, 2002.

[2] D. Edelhoff and J. A. Sorensen, "Tooth structure removal associated with various preparation designs for anterior teeth," The Journal of Prosthetic Dentistry, vol. 87, no. 5, pp. 503-509, 2002.
[3] K. Langeland, "Dentin and pulp reactions to cavity and crown preparation. Survey of literature," Annual Meeting - American Institute of Oral Biology, vol. 23, pp. 73-88, 1966.

[4] K. Langeland and L. K. Langeland, "Pulp reactions to cavity and crown preparation," Australian Dental Journal, vol. 15, no. 4, pp. 261-276, 1970.

[5] A. L. Rochette, "Attachment of a splint to enamel of lower anterior teeth," The Journal of Prosthetic Dentistry, vol. 30, no. 4, pp. 418-423, 1973.

[6] M. Wood, M. Kern, V. P. Thompson, and E. Romberg, “Tenyear clinical and microscopic evaluation of resin-bonded restorations," Quintessence International, vol. 27, no. 12, pp. 803-807, 1996.

[7] D. F. Howe and G. E. Denehy, "Anterior fixed partial dentures utilizing the acid-etch technique and a cast metal framework," The Journal of Prosthetic Dentistry, vol. 37, no. 1, pp. 28-31, 1977.

[8] G. J. Livaditis and V. P. Thompson, "Etched castings: an improved retentive mechanism for resin-bonded retainers," The Journal of Prosthetic Dentistry, vol. 47, no. 1, pp. 52-58, 1982.

[9] M. Kern, H. Knode, and J. R. Strubb, “The all-porcelain, resinbonded bridge," Quintessence International, vol. 22, no. 4, pp. 257-262, 1991.

[10] M. Kern and J. R. Strub, "Bonding to alumina ceramic in restorative dentistry: clinical results over up to 5 years," Journal of Dentistry, vol. 26, no. 3, pp. 245-249, 1998.

[11] B. Mourshed, A. Samran, A. Alfagih, A. Samran, S. Abdulrab, and M. Kern, "Anterior cantilever resin-bonded fixed dental prostheses: a review of the literature," Journal of Prosthodontics, vol. 27, no. 3, pp. 266-275, 2018.

[12] M. Kern and R. Glaser, "Cantilevered all-ceramic, resinbonded fixed partial dentures: a new treatment modality," Journal of Esthetic Dentistry, vol. 9, no. 5, pp. 255-264, 1997.

[13] M. Kern, N. Passia, M. Sasse, and C. Yazigi, "Ten-year outcome of zirconia ceramic cantilever resin-bonded fixed dental prostheses and the influence of the reasons for missing incisors," Journal of Dentistry, vol. 65, pp. 51-55, 2017.

[14] A. van Dalen, A. J. Feilzer, and C. J. Kleverlaan, "A literature review of two-unit cantilevered FPDs," The International Journal of Prosthodontics, vol. 17, no. 3, pp. 281-284, 2004.

[15] S. Wolfart and M. Kern, "A new design for all-ceramic inlayretained fixed partial dentures: a report of 2 cases," Quintessence International, vol. 37, no. 1, pp. 27-33, 2006.

[16] S. Bishti, C. Jakel, M. Kern, and S. Wolfart, "Influence of different preparation forms on the loading-bearing capacity of zirconia cantilever FDPs. A laboratory study," Journal of Prosthodontic Research, vol. 63, no. 3, pp. 347-353, 2019.

[17] M. Kern, Resin-Bonded Fixed Dental Prostheses: Minimally Invasive-Esthetic-Reliable, Quintessence Publishing, 2019.

[18] J. B. Summitt, J. W. Robbins, T. J. Hilton, R. S. Schwartz, and J. Dos Santos Jr., "Esthetic Inlays and Onlays," in Fundamentals of operative dentistry A contemporary approach, pp. 514528, Quintessence Publishing Co, 2006.

[19] S. M. Wegner, W. Gerdes, and M. Kern, "Effect of different artificial aging conditions on ceramic-composite bond strength," The International Journal of Prosthodontics, vol. 15, no. 3, pp. 267-272, 2002.

[20] M. Kern, "Single-retainer resin-bonded fixed dental prostheses as an alternative to orthodontic space closure (and to single- 
tooth implants)," Quintessence International, vol. 49, no. 10, pp. 789-798, 2018.

[21] M. Kern, "Fifteen-year survival of anterior all-ceramic cantilever resin-bonded fixed dental prostheses," Journal of Dentistry, vol. 56, pp. 133-135, 2017.

[22] M. Kern and M. Sasse, "Ten-year survival of anterior allceramic resin-bonded fixed dental prostheses," The Journal of Adhesive Dentistry, vol. 13, no. 5, pp. 407-410, 2011.

[23] M. A. Kilicarslan, P. S. Kedici, H. C. Kucukesmen, and B. C. Uludag, "In vitro fracture resistance of posterior metalceramic and all-ceramic inlay- retained resin-bonded fixed partial dentures," The Journal of Prosthetic Dentistry, vol. 92, no. 4, pp. 365-370, 2004.

[24] C. A. Mohsen, "Fracture resistance of three ceramic inlayretained fixed partial denture designs. An in vitro comparative study," Journal of Prosthodontics, vol. 19, no. 7, pp. 531-535, 2010.

[25] S. Wolfart, K. Ludwig, A. Uphaus, and M. Kern, "Fracture strength of all-ceramic posterior inlay-retained fixed partial dentures," Dental Materials, vol. 23, no. 12, pp. 1513-1520, 2007.

[26] D. Edelhoff, H. Spiekermann, and M. Yildirim, "Metal-free inlay-retained fixed partial dentures," Quintessence International, vol. 32, no. 4, pp. 269-281, 2001.

[27] S. Harder, S. Wolfart, S. Eschbach, and M. Kern, "Eight-year outcome of posterior inlay-retained all-ceramic fixed dental prostheses," Journal of Dentistry, vol. 38, no. 11, pp. 875-881, 2010.

[28] S. Wolfart, F. Bohlsen, S. M. Wegner, and M. Kern, “A preliminary prospective evaluation of all-ceramic crown-retained and inlay-retained fixed partial dentures," The International Journal of Prosthodontics, vol. 18, no. 6, pp. 497-505, 2005.

[29] M. Kern, "Clinical long-term survival of two-retainer and single-retainer all-ceramic resin-bonded fixed partial dentures," Quintessence International, vol. 36, no. 2, pp. 141147, 2005. 